(TIPS). We aim to evaluate factors influencing provision of the TIPS service and outcome in the region.

Method All cases undergoing TIPS at Freeman Hospital from December 2015 to December 2017 were identified from the interventional radiology register. Electronic records and medical notes of all patients who had TIPS performed for variceal haemorrhage were reviewed retrospectively to collect data regarding clinical demographics, length of hospital stay and outcomes.

Results A total of 46 procedures were performed; 29 for variceal haemorrhage. Two were excluded from further analysis due to non-availability of medical notes. The median age at the time of intervention was 51 (range 21-71) years and 13 $(48 \%)$ were male. Cases were referred from nine regional hospitals. The majority had alcohol related liver disease $(63 \%$ alcohol, 26\% Non-alcohol), 77\% with Child B or C cirrhosis and $85 \%$ had MELD score $>11$. A third of patients had undergone one attempt at haemostatic control with another third having had $\geq 3$ interventions prior to referral. $96 \%$ and $92 \%$ had received antibiotics and terlipressin, respectively. $56 \%$ were ITU to ITU transfers with airway protection and $52 \%$ had a Sengstaken tube in-situ [average duration of placement $17(4-48)$ hours]. Average time to transfer from referral was 18.3 hours. $57 \%$ had TIPS performed within 24 hours of arrival at the specialist centre. Although the average time to TIPS varied between weekends and weekdays, 46 and 35 hours respectively, there was no significant difference in outcome or survival $(p=0.221) .22 \%$ required inotropic support following TIPS. Average time taken for discharge from ITU after being assessed as fit for stepping down care to the ward or repatriation was $4.45(0-51)$ days. The duration of Sengstaken insertion $>24$ hours did not influence outcome or survival. $67 \%$ of patients were alive at 90 days post TIPS.

Conclusion The majority of patients received antibiotics and terlipressin during the bleeding episode consistent with good clinical practice. Time to TIPS was longer in patients admitted at weekends but with no significant difference in survival outcome. The duration of Sengstaken tube placement did not significantly influence outcome. Delays and decisions to repatriation were multifactorial, including non-availability of beds at the referring hospital, family preference to remain in centre and post TIPS complications.

\section{PTH-083 IMPACT OF HOSPITALISATION RATE FOR HCV RELATED LIVER DISEASES IN NORTH OF ITALY}

Diego Caroli*, Erik Rosa-Rizzotto, Mario Saia, Laura Scribano, Laura Peraro, Salvatore Lobello, Franca De Lazzari. St. Anthony Hospital, Padova, Italy

\subsection{6/gutjnl-2018-BSGAbstracts.239}

Background and aims Hepatitis C virus (HCV) epidemiological data in Italy is changing due to the decline of iatrogenic aetiology and the persistence of infection in populations at risk. The incidence and the prevalence of HCV disease have dropped following also the massive commitment of physicians to treat all the infected patients. However, all over the world HCV liver-related disease is the first cause of hospital admission in patients with liver diseases. We analysed the trend of hospitalisation for liver HCV-related disease in Veneto Region, North East Italy, from 2000 to 2016, in order to report the impact of HCV treatment with different therapeutic schedules.
Method This is a retrospective cohort study based on Veneto Region anonymous computerised database of hospital discharges between 2000 and 2016. All Veneto residents discharge records with principal diagnosis of hepatitis (cod. ICD9-CM: 070.41, 070.44, 070.51, 070.54, 070.70, 070.71, $571.5,571.9)$ were included in the study. We chose the principal diagnosis because it is considered the primary reason for hospital admission. The Standardised Hospitalisation Ratio (SHR) per five-year age group (ref. pop. Veneto 2008) was calculated and expressed per 100000 population.

Results In the period considered 36102 hospital admissions diagnosed with HCV have been recorded. Approximately half of patients were males (56\%). Despite their lower age $(56,1$ $\pm 7,2$ Vs. $65,1 \pm 8,3)$, they had the greatest hospitalisation rate (51,4 Vs. 37,9; OR:1.36;CI95\%:1.33-1.39; $<00.05)$. The analysis of the hospitalisation trend shows a $14 \%$ increase in the average age of patients(from $57,3 \pm 9,5$ to $65,1 \pm 9,9$ ) and a substantial decrease in hospital admissions (X2 trend: 9210,736; p <0,05). Between 2000 and 2016, there has been a $81 \%$ decline in hospital admissions (i.e. from 78,9 to 14,8 ) with a comparable decrease in both genders/sexes (ratio M:F 1.5). In 2012-2014 period we observed a plateau in the curve while in 2015-2016 the decline starts again (figure 1).

Conclusion HCV liver-related disease as cause of hospital admission is in progressive and constant decline related to the different treatment schedules available in each period. Moreover this downward trend reflects the improvement in management of advanced liver disease in outpatient settings. In the last two yrs of observation the decline starts again because of the availability of DAAs with high efficacy also in patients with advanced stage of liver disease.

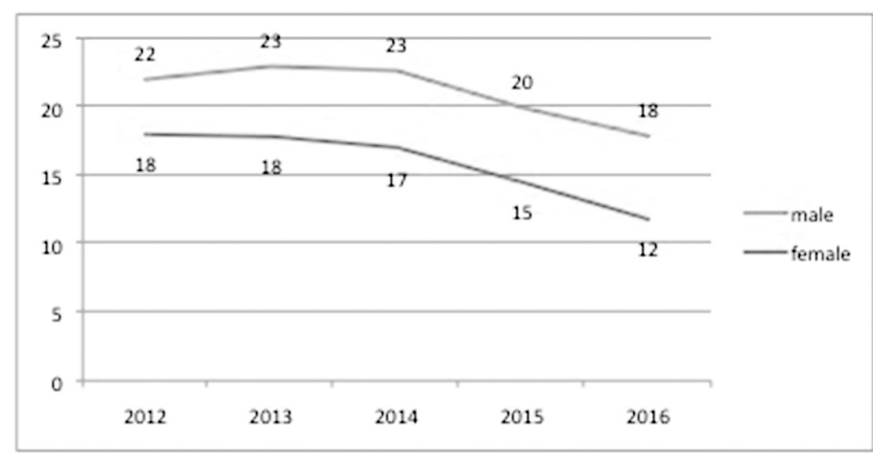

Abstract PTH-083 Figure 1

\section{PTH-084 THE EFFICACY OF TACROLIMUS AS AN ALTERNATIVE AGENT IN THE TREATMENT OF AUTOIMMUNE HEPATITIS}

Geraldine Carroll*, Gemma Wasson, Neil McDougall, Roger McCorry, lan Cadden, Jonathan Cash. Royal Victoria Hospital, Belfast, UK

\subsection{6/gutjnl-2018-BSGAbstracts. 240}

Introduction Autoimmune hepatitis (AIH) is a chronic, inflammatory liver condition which, if untreated, can Result in liver cirrhosis. Current BSG guidelines recommend corticosteroids and azathioprine as first line therapy, with the option of switching to mycophenolate if azathioprine is not tolerated. Tacrolimus has been identified as a potential third line treatment strategy. Our aim was to review the outcomes of 
patients with a diagnosis of AIH who required the addition of tacrolimus as a third line agent.

Methods The tacrolimus database for the Regional Liver Unit, Royal Victoria Hospital was reviewed to identify all patients with AIH who had been treated with tacrolimus from Jan 2010 until August 2017. Records were cross referenced with the diagnostic coding department. Demographic details, indications for tacrolimus therapy, clinical and biochemical outcomes were recorded.

Results 30 patients were identified (24 (80\%) female, mean age 40.7 years, range $19-81$ years). 27 of the 30 patients were initially treated with azathioprine of whom 21 (78\%) discontinued treatment due to adverse effects including blood dyscrasias and $6(22 \%)$ were switched to tacrolimus due treatment failure. Three of 30 patients were started on tacrolimus instead of azathioprine or mycophenolate. Two of these patients had previous episodes of pancytopenia at the time of commencing treatment for $\mathrm{AIH}$ and azathioprine/mycophenolate were excluded as a treatment option. One of the patients was commenced on prednisolone and tacrolimus without another steroid sparing agent trailed for other reasons. 26 $(87 \%)$ of 30 patients remain on tacrolimus, of whom 11 $(42 \%)$ had normalisation of transaminases and a further 12 $(46 \%)$ had improvement of transaminases. Liver function tests in the $3(11.5 \%)$ remaining patients were deranged but static. Of note all three had established cirrhosis at the time of AIH diagnosis. Of the four whose tacrolimus therapy was discontinued, two stopped due to side effects, 1 is deceased (not tacrolimus related) and one stopped due to commencing infliximab for IBD.

Conclusions Tacrolimus is a safe and well tolerated treatment for AIH when first line therapy has failed. In the cohort observed, only $6 \%$ failed to tolerate tacrolimus and biochemical parameters were improved or normalised in $88 \%$ of patients who remained on tacrolimus therapy.

\section{PTH-085 USING ELF TESTS IN PRIMARY AND SECONDARY CARE TO IDENTIFY PATIENTS WITH ADVANCE FIBROSIS}

Charlotte Cook*, Alison Burridge, Janisha Patel. University Hospital Southampton, Southampton, UK

\subsection{6/gutjpl-2018-BSGAbstracts.241}

Introduction In 2016 NICE NAFLD guidelines recommended Enhance Liver Fibrosis (ELF) as a validated test to assess advanced liver fibrosis.

This study aims

ELF tests were performed, 166 from by GP practices and 175 by UHS hepatology service. 89 were from other organisations. to assess the relationship with standard investigations used in a hepatology clinic for NAFLD patients (including transient elastography) and the newly commissioned ELF test in a real world setting.

Methods The study looked at all new patients diagnosed with NAFLD via the hepatology outpatient clinic at University Hospital Southampton between November 2016 and May 2017.

Each patient had their demographics (age, gender, weight) and comorbidities (diabetes, hypertension, dyslipidaemia) assessed. Transient elastography, USS and ELF Result (when performed) were reviewed.

The ELF was categorised as no/mild fibrosis if $<7.5$, moderate fibrosis if $\geq 7.5$ and $\leq 10.5$, advanced fibrosis if $\geq 10.51$.
The ELF tests requested over the same period were analysed review the source of the request.

Results 175 people were diagnosed with NAFLD through hepatology clinic. All patients had transient elastography performed. The mean LSM Result was 14.6 (range 3.3-75), with a mean CAP score of 313.6 (range 100-400). Of 175 new diagnoses, 101 patients were male (mean weight $99 \mathrm{~kg}$, median age 52 years) and 74 were female $(87.1 \mathrm{~kg}$ and 57 years).

69 patients had type 2 diabetes mellitus, 74 had hypertension and 66 had dyslipidaemia. 30 patients had all 3 (16 were female with a mean weight $96.5 \mathrm{~kg}$ and mean LSM $18.5 \mathrm{kPa}$ and 14 were male, $100.7 \mathrm{~kg}$ and $19.3 \mathrm{kPa}$ ).

With transient elastography, 59 patients had LSM $<6 \mathrm{kPa}$, 68 were $6.1-12 \mathrm{kPa}, 23$ were $12.1-20 \mathrm{kPa}$ and $25>20.1 \mathrm{kPa}$. This suggested $48(27 \%)$ patients had advanced fibrosis or cirrhosis. The remaining 127 (73\%) patients did not require hepatology review.

430The GP cohort had 18 (11\%) patients with an ELF test $\geq 10.5$ requiring hepatology review. 36 (21\%) patients seen in the hepatology clinic had an ELF $>10.51$, the remaining 139 (79\%) patient did not require a hepatology review.

Conclusions This study looked at the cohort of new diagnoses of NAFLD in a teaching hospital using standard tests and ELF score. Currently, access to transient elastography is secondary care based. The preliminary data in this study shows that the ELF test is a good first line investigation for GPs suspecting NAFLD in a patient with type 2 diabetes and obesity or incidental finding of fatty liver. It promotes the need to look beyond the routine liver panel test and identify the aetiology of liver disease and assess extent of liver fibrosis; in turn, to generate appropriate secondary care referrals and incorporate efficiency. Further assessment of the use of ELF in this setting continues

\section{PTH-086 VIRTUAL HEPATITIS B CLINICS SIGNIFICANTLY IMPROVE COST AND CLINICAL EFFECTIVENESS}

Douglas Corrigall* Sarah Fairclough, Linda Porter, Katrina White, Denise Killworth, Gavin Wright. Basildon and Thurrock University Hospital, Basildon, UK

10.1136/gutjnl-2018-BSGAbstracts.242

Introduction Chronic Hepatitis B (HBV) infection requires regular hepatology review to assess for potential flares, need for therapy and/or progression to advanced liver disease and its complications. The majority of patients are chronic carriers who feel perfectly well and can find repeated clinic visits difficult, unnecessary and impinge on other commitments (e.g. employment, social and child care). As a Result there is a high rate of missed appointments and or patients lost to follow up, with the potential for missed viral flares. A nurse-led virtual clinic allows remote monitoring of these patients as recommended by national and international guidelines. ${ }^{1} 2$

Methods Patients with stable chronic HBV suitable for telephone clinic follow-up were identified. The criteria used were: not on/received HBV therapy, no significant comorbidities or risk factors for liver disease, low viral load $(<2000 \mathrm{iu} / \mathrm{ml}), \mathrm{F} 0 /$ 1 disease, low and stable alpha-fetoprotein (AFP), unremarkable imaging, and no transaminitis/native liver derangement.

Over a 2.5 year period from 01.02.2014, patients meeting the criteria were offered 6-monthly virtual clinic review. Patients had surveillance blood test within 4 weeks of their 